\title{
MicroRNA-137 has a suppressive role in liver cancer via targeting EZH2
}

\author{
SHICHANG CUI $^{1}$, YANLEI SUN ${ }^{1}$, YANG LIU ${ }^{2}$, CHENGBIAO LIU ${ }^{1}$, \\ JINBAO WANG $^{1}$, GUANG HAO ${ }^{1}$ and QIDONG SUN ${ }^{1}$ \\ Departments of ${ }^{1}$ General Surgery and ${ }^{2}$ Obstetrics and Gynecology, Central Hospital of Linyi, \\ Linyi, Shandong 276400, P.R. China
}

Received September 13, 2016; Accepted June 23, 2017

DOI: $10.3892 / \mathrm{mmr} .2017 .7828$

\begin{abstract}
A variety of microRNAs (miRs) have been demonstrated to be associated with the development and malignant progression of human cancer; however, the regulatory mechanism of miR-137 underlying hepatocellular carcinoma (HCC) growth and metastasis still remains to be fully revealed. In the present study, reverse transcription-quantitative polymerase chain reaction and western blot were used to examine mRNA and protein expression. MTT assay, wound healing assay and Transwell assay were performed to determine cell proliferation, migration and invasion. Luciferase reporter assay was conducted to confirm the targeting relationship. miR-137 was significantly downregulated in HCC tissues compared to adjacent normal tissues. Low expression of miR-137 was significantly associated with lymph node metastasis, vein invasion, advanced clinical stage and poor prognosis in HCC. In addition, miR-137 was also downregulated in several liver cancer cell lines compared with normal liver epithelial cells. Overexpression of miR-137 led to a significant reduction in cell proliferation, migration and invasion of $\mathrm{HepG} 2$ cells. Enhancer of zeste 2 polycomb repressive complex 2 subunit (EZH2) was further identified as a direct target gene of miR-137, and the protein expression of EZH2 was negatively regulated by miR-137 in HepG2 cells. Additionally, EZH2 was significantly upregulated in HCC tissues and liver cancer cell lines. Furthermore, overexpression of EZH2 significantly eliminated the inhibitory effects of miR-137 on the malignant phenotypes of HepG2 cells. Therefore, the findings suggest that miR-137 may have a suppressive role in HCC growth and metastasis via targeting EZH2.
\end{abstract}

Correspondence to: Professor Qidong Sun, Department of General Surgery, Central Hospital of Linyi, 17 Jiankang Road, Yishui, Linyi, Shandong 276400, P.R. China

E-mail: sunqidong2015@sina.com

Key words: liver cancer, hepatocellular carcinoma, microRNA, enhancer of zeste homologue 2, proliferation, migration, invasion

\section{Introduction}

Hepatocellular carcinoma (HCC), one of the most common human malignancies, causes a large amount of mortalities worldwide, and its incidence has increased rapidly in recent years $(1,2)$. Despite great efforts in diagnosis and treatment, including surgical resection, radiotherapy and chemotherapy, the prognosis of patients with $\mathrm{HCC}$ remains poor, predominantly due to the recurrence and metastasis (2). Therefore, revealing the molecular mechanism underlying HCC growth and metastasis is urgently required, which may help to develop targeted treatment strategies.

MicroRNAs (miRs) are a type of short non-coding RNA, and generally function as negative regulators of gene expression through directly binding to the 3 -untranslational region (3'-UTR) of their target genes, resulting in mRNA degradation or translation inhibition $(3,4)$. In the last decade, miRs have been demonstrated to participate in various biological processes, including cell survival, proliferation, differentiation, apoptosis and motility (3,5). Furthermore, various miRs have been reported to act as oncogenes or tumor suppressors in human malignancies including HCC (6-8). For instance, miR-212, downregulated in HCC tissues, suppresses tumor growth by inhibition of forkhead box A1 (9). miR-98 suppresses cell proliferation, migration and invasion in HCC by targeting the collagen triple helix repeat containing 1 oncogene (10). These miRs associated with the development and progression of HCC may become important therapeutic targets or reagents for this disease.

Recently, miR-137 was reported to function as a tumor suppressor in some common human cancer types $(11,12)$. Shen et al (11) reported that miR-137 inhibited tumor growth and sensitize chemosensitivity to paclitaxel and cisplatin in lung cancer. Liang et al (12) reported that downregulation of miR-137 promoted cell proliferation in pediatric high-grade gliomas. Furthermore, the suppressive role of miR-137 in HCC has gradually been elucidated (13). Liu et al (14) reported that miR-137 suppresses tumor growth and metastasis in HCC by targeting AKT serine/threonine kinase 2. Gao et al (15) reported that the suppressive effects of miR-137 on HCC cell proliferation and metastasis were regulated by cell division cycle 42 . As one miR has multiple target genes, there may also be other important targets of miR-137 in HCC (3). The current study aimed to investigate the molecular mechanism 
of miR-137 in regulating HCC cell proliferation, migration and invasion.

\section{Materials and methods}

Tissue collection. The current study was approved by the Ethics Committee of Central Hospital of Linyi (Linyi, China). A total of 89 primary HCC tissues and adjacent non-tumor tissues were collected from patients at the Central Hospital of Linyi between September 2010 and June 2012, and informed consent was obtained from all participants. No patients received radiation therapy or chemotherapy prior to surgical resection. Tissues were immediately snap-frozen in liquid nitrogen following surgical resection, and stored in liquid nitrogen prior to use. The clinical information of patients involved in this study is summarized in Table I.

Cell culture and transfection. Human normal liver epithelial cell line THLE-3 and four liver cancer cell lines including HepG2, Hep3B HCCLM3 and SMCC7721 were purchased from Cell Bank of Chinese Academy of Sciences (Shanghai, China). Cells were cultured in Dulbecco's modified Eagle's medium (DMEM; Thermo Fisher Scientific, Inc., Waltham, MA, USA) supplemented with $10 \%$ fetal bovine serum (FBS; Thermo Fisher Scientific, Inc.) in a $37^{\circ} \mathrm{C}$ humidified atmosphere with $5 \% \mathrm{CO}_{2}$. For cell transfection, HepG2 cells were transfected with scramble miR mimic (miR-NC) (CmiR0001-MR04), miR-137 mimic (HmiR0011-MR04), negative control (NC) inhibitor (CmiR-AN0001-SN) miR-137 inhibitor (HmiR-AN0175-SN-10) (all purchased from Guangzhou Fulengen Co., Ltd., Guangzhou, China) or co-transfected with miR-137 mimic and pcDNA3.1-enhancer of zeste 2 polycomb repressive complex 2 subunit (EZH2) expression plasmid (Yearthbio, Changsha, China) using Lipofectamine 2000 (Thermo Fisher Scientific, Inc.), according to the manufacturer's instructions.

Reverse transcription-quantitative polymerase chain reaction $(R T-q P C R)$. Total RNA was extracted from $1 \times 10^{7}$ cells (THLE-3 cells and the liver cancer cell lines) using TRIzol Reagent (Thermo Fisher Scientific, Inc.). Total RNA was then converted into cDNA using PrimeScript First Strand cDNA Synthesis kit (Takara Bio, Inc., Otsu, Japan). For miR expression, RT-qPCR was performed using miRNA Q-PCR Detection kit (GeneCopoeia, Inc., Rockville, MD, USA) according to the manufacturer's instructions on an ABI 7500 thermocycler (Thermo Fisher Scientific, Inc.). U6 was used as a reference gene internal control for miR expression. U6 (HmiRQP9001) and miR-137 (HmiRQP0175) primers were purchased from Guangzhou Fulengen Co., Ltd. For mRNA expression detection, RT-qPCR was performed using SYBR-Green I Real-Time PCR kit (Biomics Biotechnologies Co., Ltd., Nantong, China). The primer sequences for EZH2 were as follows: Forward, 5'-AATCAGAGTACATGCGAC TGAGA-3' and reverse, 5'-GCTGTATCCTTCGCTGTT TCC-3'. The primer sequences for GAPDH were as follows: Forward, 5'-CTGGGCTACACTGAGCACC-3' and reverse, 5'-AAGTGGTCGTTGAGGGCAATG-3'. The PCR reaction system was $0.33 \mu \mathrm{l}$ cDNA solution, $10 \mu \mathrm{l}$ SYBR-Green I mix, $2 \mu \mathrm{l}$ primers and $7.67 \mu \mathrm{l} \mathrm{H}_{2} \mathrm{O}$ were mixed to obtain a final
Table I. Association between miR-137 expression and clinicopathological characteristics in hepatocellular carcinoma.

\begin{tabular}{|c|c|c|c|}
\hline Variable & $\begin{array}{l}\text { High miR-137 } \\
\text { expression } \\
(n=47)\end{array}$ & $\begin{array}{c}\text { Low miR-137 } \\
\text { expression } \\
\quad(n=42)\end{array}$ & P-value \\
\hline Age & & & 0.399 \\
\hline$<55$ & 18 & 20 & \\
\hline$\geq 55$ & 29 & 22 & \\
\hline Sex & & & 1 \\
\hline Male & 32 & 28 & \\
\hline Female & 15 & 14 & \\
\hline $\begin{array}{l}\text { Tumor number } \\
\text { Single } \\
\text { Multiple }\end{array}$ & & $\begin{array}{c}35 \\
7\end{array}$ & 0.211 \\
\hline $\begin{array}{l}\text { Histologic grade } \\
\text { Well-moderate } \\
\text { Poor }\end{array}$ & 19 & $\begin{array}{l}29 \\
13\end{array}$ & 0.384 \\
\hline $\begin{array}{l}\text { Nodal meta } \\
\text { Present } \\
\text { Absent }\end{array}$ & $\begin{array}{l}25 \\
22\end{array}$ & $\begin{array}{l}11 \\
31\end{array}$ & $0.017^{\mathrm{a}}$ \\
\hline $\begin{array}{l}\text { Vessel invasion } \\
\text { Present } \\
\text { Absent }\end{array}$ & $\begin{array}{l}29 \\
18\end{array}$ & $\begin{array}{l}12 \\
30\end{array}$ & $0.003^{\mathrm{b}}$ \\
\hline $\begin{array}{l}\text { Hepatitis B } \\
\text { infection }\end{array}$ & & & 0.226 \\
\hline $\begin{array}{l}\text { Present } \\
\text { Absent }\end{array}$ & $\begin{array}{l}32 \\
15\end{array}$ & $\begin{array}{r}34 \\
8\end{array}$ & \\
\hline $\begin{array}{l}\text { Clinical T stage } \\
\text { T1-2 } \\
\text { T3-4 }\end{array}$ & $\begin{array}{l}27 \\
20\end{array}$ & $\begin{array}{l}11 \\
31\end{array}$ & $0.005^{\mathrm{b}}$ \\
\hline
\end{tabular}

${ }^{\mathrm{a}} \mathrm{P}<0.05,{ }^{\mathrm{b}} \mathrm{P}<0.01 . \mathrm{miR}-137$, microRNA-137.

reaction volume of $20 \mu \mathrm{l}$. The reaction condition were $95^{\circ} \mathrm{C}$ for $5 \mathrm{~min}$, followed by 40 cycles of denaturation at $95^{\circ} \mathrm{C}$ for $15 \mathrm{sec}$ and annealing/elongation step at $60^{\circ} \mathrm{C}$ for $30 \mathrm{sec}$. The relative expression was analyzed by the $2^{-\Delta \Delta \mathrm{Cq}}$ method (16).

Western blot analysis. Cells $\left(1 \times 10^{7}\right)$ were lysed with ice-cold radioimmunoprecipitation assay lysis buffer (Beyotime Institute of Biotechnology, Haimen, China). Proteins were collected, quantified using a bicinchoninic acid assay and separated (50 $\mu \mathrm{g} /$ lane) by $10 \%$ SDS-PAGE (Beyotime Institute of Biotechnology). Proteins were transferred onto polyvinylidene difluoride (PVDF) membrane (Thermo Fisher Scientific, Inc.). The PVDF membrane was incubated with phosphate buffered saline (PBS; Thermo Fisher Scientific, Inc.) containing 5\% non-fat milk overnight at $4^{\circ} \mathrm{C}$, and then incubated with the polyclonal mouse anti-human EZH2 antibody (1:100; cat. no. ab168764; Abcam, Cambridge, MA, USA) and polyclonal mouse anti-human GAPDH antibody (1:50; cat. no. ab8245; Abcam) at room temperature for $3 \mathrm{~h}$. After washing with PBS-Tween three times, the membrane was incubated with 
goat polyclonal anti-mouse HRP-conjugated secondary antibody (1:5,000; cat. no. ab97040; Abcam) at room temperature for $1 \mathrm{~h}$. The enhanced chemiluminescence system (Thermo Fisher Scientific, Inc.) was used to detect the immunoreactive bands. The protein expression was measured using Image Pro Plus software (Media Cybernetics, Inc., Rockville, MD, USA). GAPDH was used as the internal reference.

Cell proliferation analysis. HepG2 cell suspension $\left(5 \times 10^{4}\right.$ cells/well) was plated in a 96-well plate, and cultured for $12,24,48$ or $96 \mathrm{~h}$. Subsequently, MTT $(10 \mu \mathrm{l}, 5 \mathrm{mg} / \mathrm{ml})$ was added, and cells were then incubated at $37^{\circ} \mathrm{C}$ for $4 \mathrm{~h}$. The supernatant was removed, and $100 \mu$ ldimethyl sulfoxide was added into each well. The absorbance at $570 \mathrm{~nm}$ was determined using the Model 680 Microplate Reader (Bio-Rad Laboratories, Inc., Hercules, CA, USA).

Cell migration analysis. HepG2 cells were cultured to confluence, and a wound was created with a plastic scriber. Then, cells were washed and incubated in serum-free DMEM at $37^{\circ} \mathrm{C}$ for $24 \mathrm{~h}$. Subsequently, the medium was replaced with DMEM with $10 \% \mathrm{FBS}$, and then cultured at $37^{\circ} \mathrm{C}$ for $24 \mathrm{~h}$. Cells were observed under a Nikon microscope (Nikon Corporation, Tokyo, Japan).

Cell invasion analysis. A 24-well Transwell chamber (Chemicon; Merck KGaA, Darmstadt, Germany) with a layer of Matrigel was used to perform Transwell assays. A HepG2 cell suspension $\left(5 \times 10^{4}\right.$ cells) was added in the upper chamber, and DMEM containing 10\% FBS was added into the lower chamber. After incubation for $24 \mathrm{~h}$, non-invading cells on the interior of the inserts were removed using a cotton-tipped swab. Cells on the lower surface of the membrane was stained with $0.5 \%$ gentian violet at room temperature for $10 \mathrm{~min}$, and then rinsed by water, and air-dried. Inyading cells were counted under a Nikon microscope.

Luciferase reporter assay. Targetscan 7.0 (www.targetscan. org) was used to predicate the putative targets of miR-137, according to the manufacturer's instructions. According to the manufacturer's instructions, the QuickChange Site-Directed Mutagenesis kit (Stratagene; Agilent Technologies, Inc., Santa Clara, CA, USA) was used to construct the mutant type (MT) EZH2 3' untranslated region (3'UTR) lacking complementarity with the miR-137 seed sequence. The wild-type (WT) or MT of EZH2 3'UTR was cloned into the downstream of the firefly luciferase-coding region of pMIR-GLO ${ }^{\mathrm{TM}}$ Luciferase vector (Promega Corporation, Madison, WI, USA). The cloning procedure was performed by Yearthbio. HepG2 cells were co-transfected using Lipofectamine 2000 with the WT- or MT-EZH2-3'UTR luciferase reporter plasmid, and miR-NC or miR-137 mimic, respectively. The luciferase activity was detected after transfection for $48 \mathrm{~h}$ using the Dual Luciferase Reporter Assay system (Promega Corporation), according to the manufacturer's instruction.

Statistical analysis. Data are expressed as the mean \pm standard deviation of three independent experiments. SPSS 19.0 (IBM Corp., Armonk, NY, USA) was used to perform statistical analysis. The association between miR-137 expression and clinical characteristics in HCC were analyzed using $\chi^{2}$ test. The

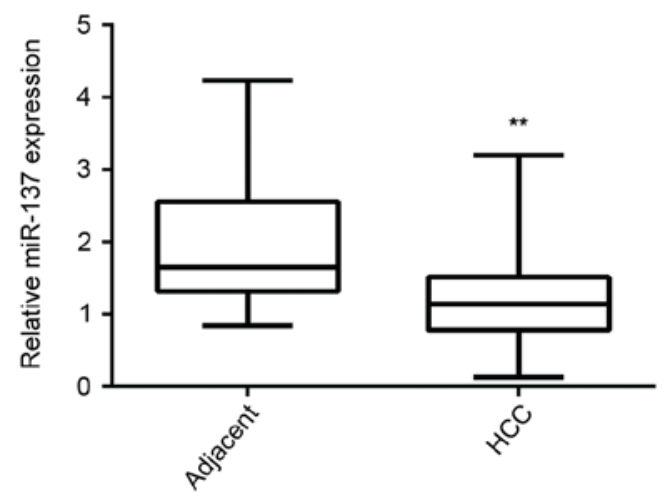

B

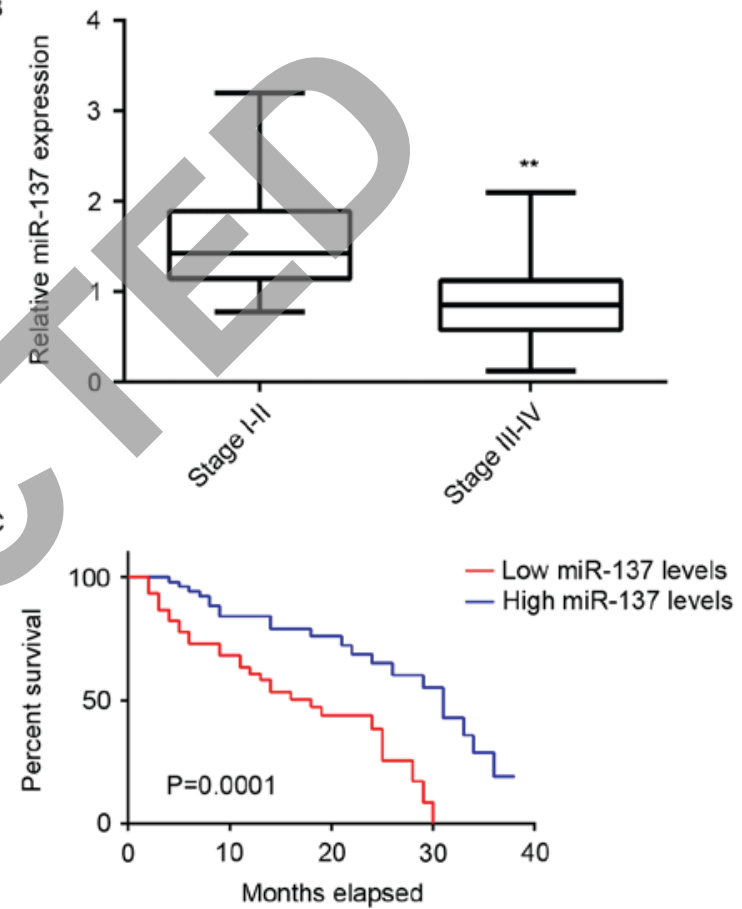

Figure 1. miR-137 expression in HCC. (A) RT-qPCR was used to examine miR-137 expression in HCC tissues compared with adjacent non-tumor tissues. ${ }^{* *} \mathrm{P}<0.01$ vs. adjacent. (B) RT-qPCR was used to examine miR-137 expression in stage III-IV HCC tissues compared with stage I-II HCC tissues. ${ }^{* *} \mathrm{P}<0.01$ vs. stage I-II. (C) Patients with HCC with low miR-137 expression had shorter survival time, when compared with those with high expression of miR-137. RT-qPCR, reverse transcription-quantitative polymerase chain reaction; miR, microRNA; HCC, hepatocellular carcinoma.

Kaplan-Meier estimator was used for survival analysis. The difference between two groups was analyzed using Student's t-test. The difference among more than two groups was analyzed using analysis of variance and Tukey post hoc test. Pearson correlation analysis was used to analyze the correlation between miR-137 and EZH2 mRNA levels in HCC tissues. $\mathrm{P}<0.05$ was considered to indicate a statistically significant difference.

\section{Results}

Downregulation of miR-137 is associated with malignant progression and poor prognosis in $\mathrm{HCC}$. To study the regulatory mechanism of miR-137 in HCC, its expression levels were initially examined in HCC tissues and adjacent non-tumor tissues. RT-qPCR data demonstrated that miR-137 was significantly downregulated in HCC tissues compared with adjacent 
A

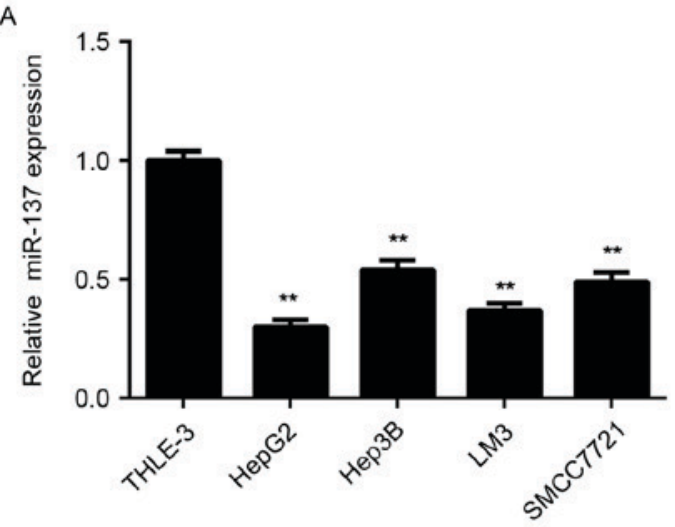

C

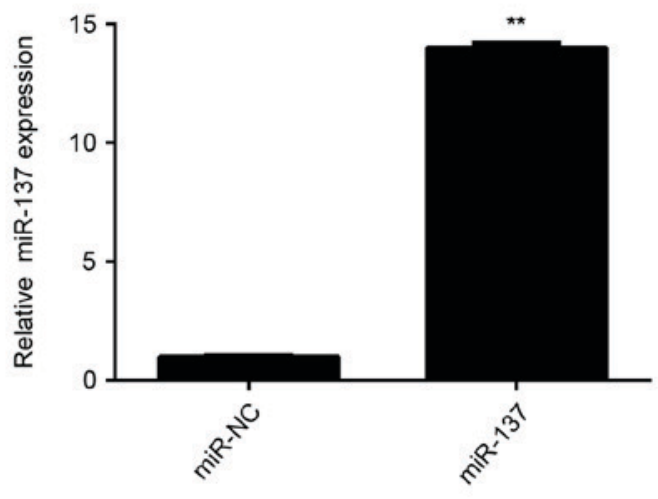

D

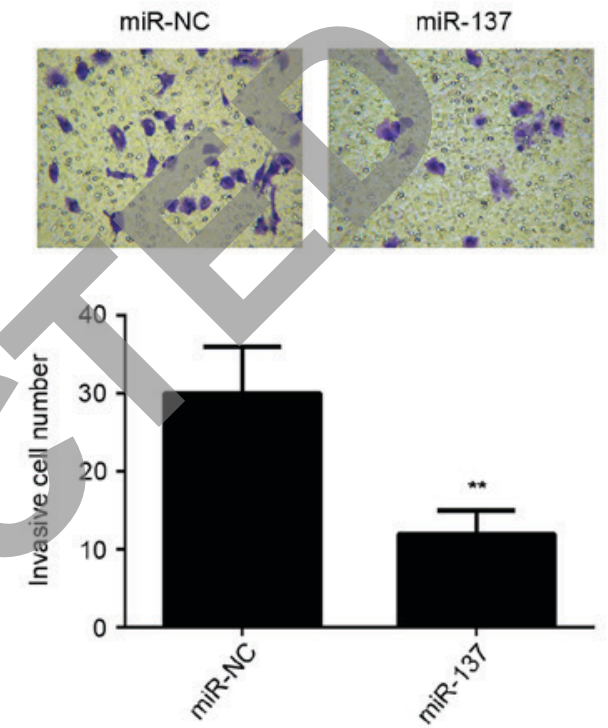

st

E
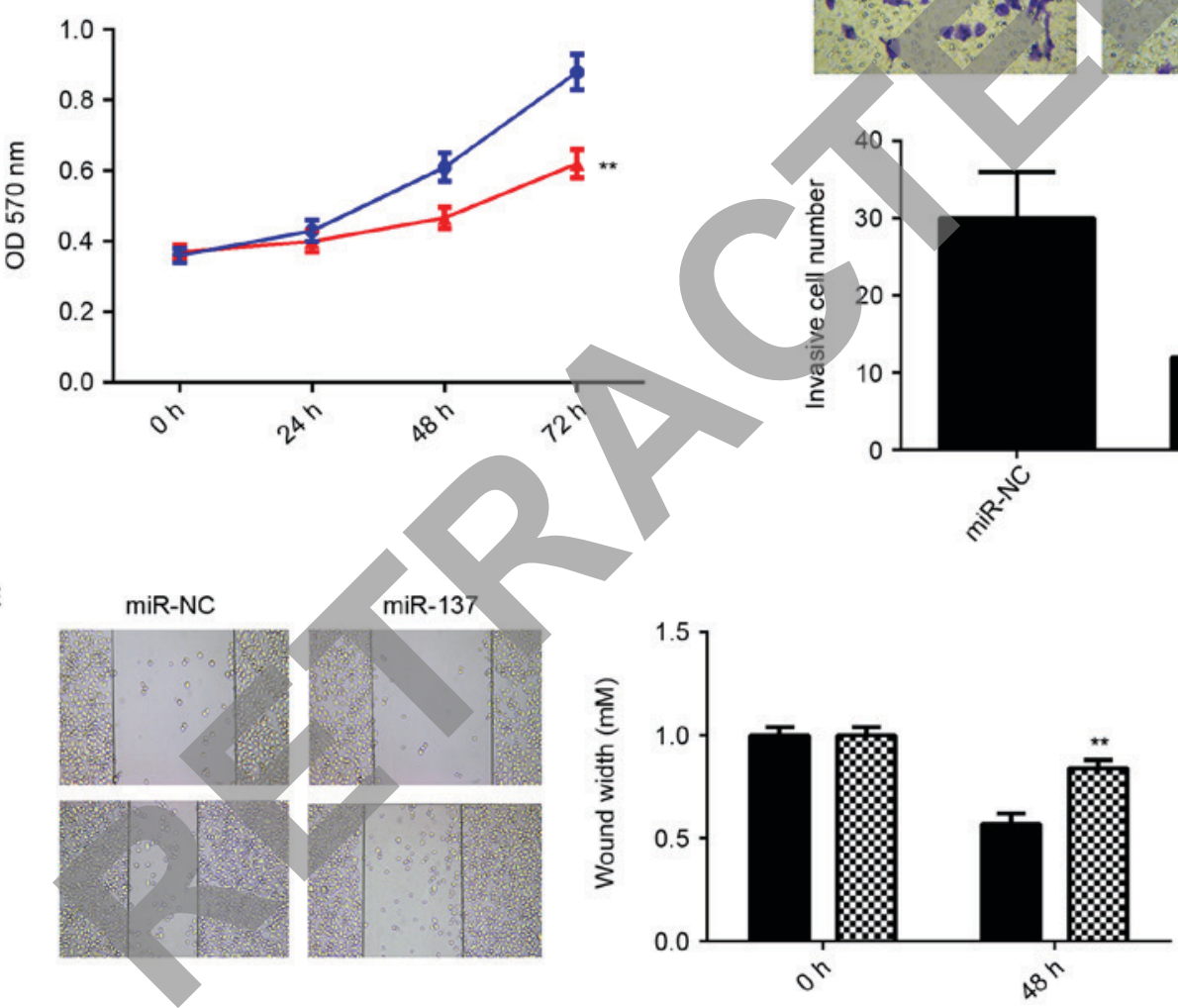

miR-NC

miR-137

Figure 2. Effect of miR-137 mimic on HepG2 cells. (A) RT-qPCR was used to examine the miR-137 expression in liver cancer cell lines compared with normal liver epithelial THLE-3 cells. * P $<0.01$ vs. THLE-3. (B) HepG2 cells were transfected with miR-137 mimic or miR-NC and RT-qPCR was used to examine the miR-137 expression. ${ }^{* *} \mathrm{P}<0.01$ vs. miR-NC. (C) MTT assay, (D) Transwell assay (magnification, $\left.\mathrm{x} 400\right)$ and (E) wound healing assay (magnification, $\mathrm{x} 40$ ) were conducted to examine the cell proliferation, invasion and migration of HepG2 cells, respectively. ${ }^{* *} \mathrm{P}<0.01$ vs. miR-NC. RT-qPCR, reverse transcription-quantitative polymerase chain reaction; miR, microRNA; miR-NC, negative control scramble miR; OD, optical density.

non-tumor tissues (Fig. 1A). Additionally, the expression of miR-137 was further lower in HCC at high stage (III-IV) compared with that in HCC at low stage (I-II; Fig. 1B). These findings suggest that the decreased expression of miR-137 may contribute to the malignant progression of HCC. To further confirm these findings, the association between the miR-137 expression and clinical characteristics in HCC was examined. Using the mean value of miR-137 expression as a cutoff, the HCC patients were divided into a high miR-137 expression group and low miR-137 expression group. Low expression of miR-137 was significantly associated with vein invasion, lymph node metastasis and advanced clinical stage in HCC, but not associated with age, sex, tumor number, histologic grade, or HPV infection (Table I). Taken these findings together, we suggest that downregulation of miR-137 is involved in HCC progression. Subsequently, the association between miR-137 expression and overall survival time of patients with HCC was analyzed. As presented in Fig. 1C, the patients with HCC with low expression of miR-137 had shorter survival time compared with those with high miR-137 levels. These findings suggest that downregulation of miR-137 is associated with poor prognosis in $\mathrm{HCC}$. 
A

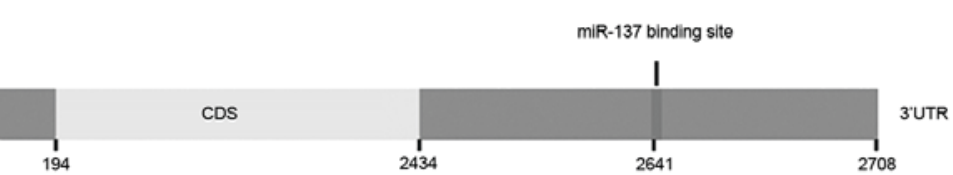

\begin{tabular}{|c|c|c|c|c|c|c|c|}
\hline & Predicted consequential paring of target region (top) and miR $\mathrm{NA}$ (bottom) & Site type & Context++ score & $\begin{array}{l}\text { Context+t score } \\
\text { percentlle }\end{array}$ & $\begin{array}{l}\text { Weighted } \\
\text { contextit+ score }\end{array}$ & $\begin{array}{l}\text { Conserved branch } \\
\text { length }\end{array}$ & $P_{c r}$ \\
\hline $\begin{array}{l}\text { Position } 207-213 \text { of EZH2 } 33^{\prime} \text { UTR } \\
\text { hsa-miR-137 }\end{array}$ & $\begin{array}{c}\text {.UACCAGUGAAUUUUU-GCAAUAAU... } \\
\text { III IIIIIII } \\
\text { GAUGCGCAUAAGAAUUCGUUAUU }\end{array}$ & 7 mer-A1 & -0.26 & 90 & -0.26 & 3.984 & 0.79 \\
\hline
\end{tabular}

B
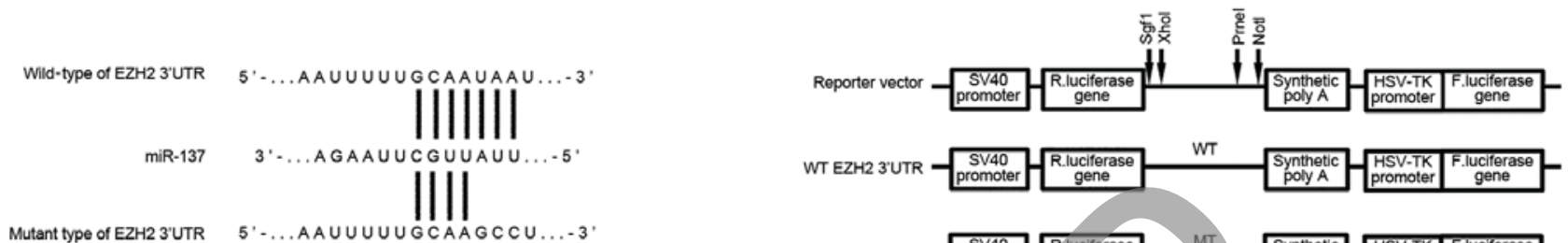

Mutant type of EZH2 3'UTR 5 ' .... A A UU UU U G CAAGCCU...-3

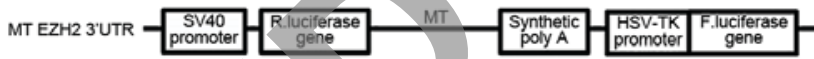

C

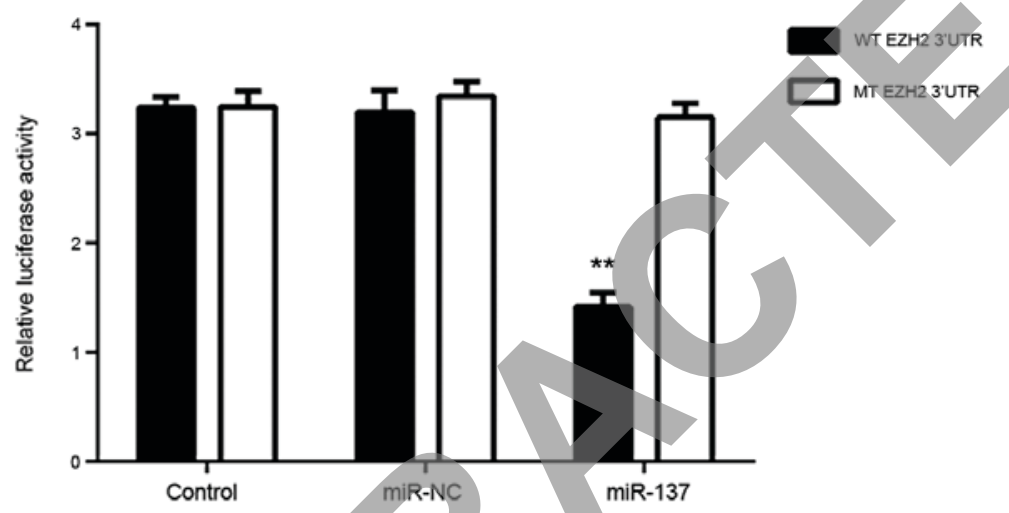

Figure 3. Effect of miR-137 on EZH2 using luciferase assay. (A) EZH2 was predicated to be a putative target of miR-137. (B) WT-EZH2-3'UTR and MT-EZH2-3'UTR luciferase reporter plasmid were generated. (C) Luciferase activity was measured reduced in cells co-transfected with miR-137 mimic and WT-EZH2-3'UTR luciferase reporter plasmid or MT-EZH2-3'UTR luciferase reporter plasmid. ${ }^{* *} \mathrm{P}<0.01$ vs. control. EZH2, enhancer of zeste 2 polycomb repressive complex 2 subunit; UTR, untranslated region; CDS, coding sequence; miR, microRNA; R. luciferase, Renilla luciferase; F. luciferase, firefly luciferase; WT, wild-type; MT, mutant; miR-NC, negative control scramble miR.

miR-137 inhibits HCC cell proliferation and invasion. To further investigate the underlying mechanism, in vitro experiments using liver cancer cell lines were performed. RT-qPCR data showed that the miR-137 levels were significantly reduced in HepG2, Hep3B, LM3 and SMCC7721 cell lines compared with THLE-3 normal liver epithelial cells (Fig. 2A). HepG2 cells were then transfected with miR-137 mimic or miR-NC as the control group. Following transfection, the miR-137 levels were examined using RT-qPCR. As presented in Fig. 2B, the miR-137 levels were significantly increased in HepG2 cells transfected with miR-137 mimic compared with the miR-NC group. Further studies demonstrated that overexpression of miR-137 caused a significant reduction in cell proliferation, migration and invasion compared with the miR-NC group (Fig. 2C-E). These data suggest that miR-137 has a suppressive role in $\mathrm{HCC}$ growth and metastasis.

EZH2 is a direct target of miR-137 in HepG2 cells. Targetscan software was used to predict that EZH2 was a putative target of miR-137 (Fig. 3A). To confirm their targeting relationship, the WT-EZH2-3'UTR or MT-EZH2-3'UTR luciferase reporter plasmids were generated (Fig. 3B). HepG2 cells were then co-transfected with WT- or MT-EZH2-3'UTR luciferase reporter plasmid, and miR-NC or miR-137 mimic. As demonstrated in Fig. 3C, the luciferase activity was significantly reduced in cells co-transfected with miR-137 mimic and WT-EZH2-3'UTR luciferase reporter plasmid when compared with the control group; the same effect was not observed in cells co-transfected with miR-137 mimic and MT-EZH2-3'UTR. Accordingly, these results indicated that miR-137 is a direct target gene of miR-137 in HepG2 cells.

Effects of miR-137 on the protein expression of EZH2 were also determined. The protein levels of EZH2 were significantly reduced in the miR-137 group compared to miR-NC group, indicating that overexpression of miR-137 downregulates EZH2 expression (Fig. 4A). To further confirm these findings, HepG2 cells were transfected with miR-137 inhibitor, or NC inhibitor as the control group. RT-qPCR data demonstrated that the miR-137 levels were downregulated in miR-137 inhibitor group compared to NC inhibitor group (Fig. 4B). Additionally, the miR-137 inhibitor significantly increased the protein expression of EZH2 in HepG2 cells (Fig. 4C). Accordingly, these results indicated that miR-137 negatively regulates the protein expression of the target gene, EZH2, in HepG2 cells. $E Z H 2$ is upregulated in $\mathrm{HCC}$ and inversely correlated 
A

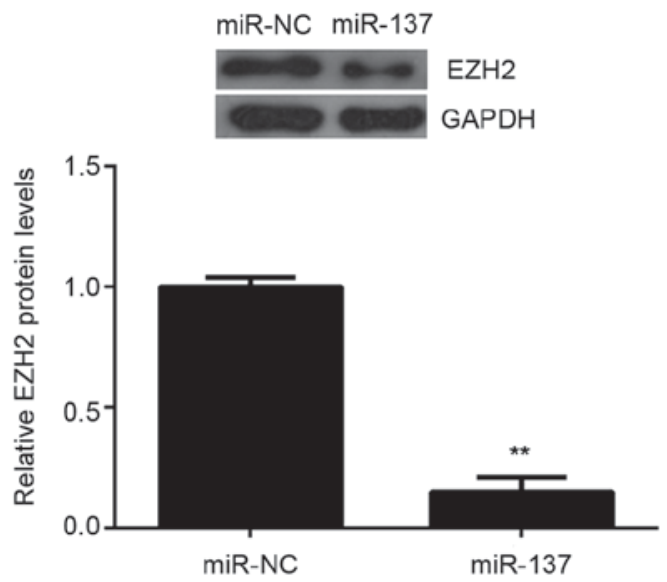

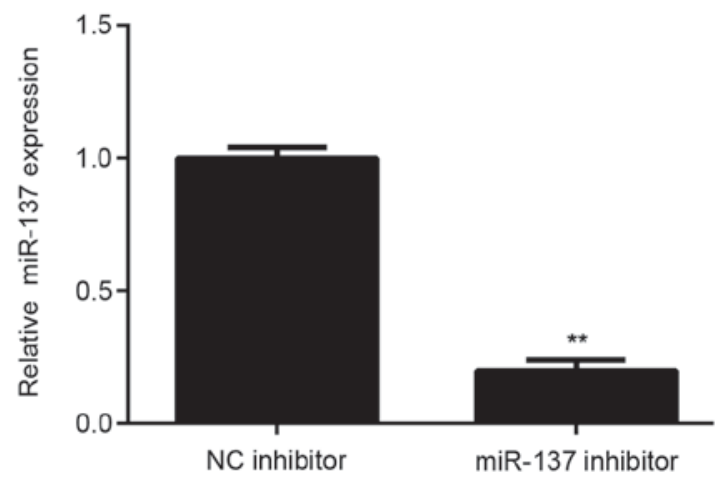

C

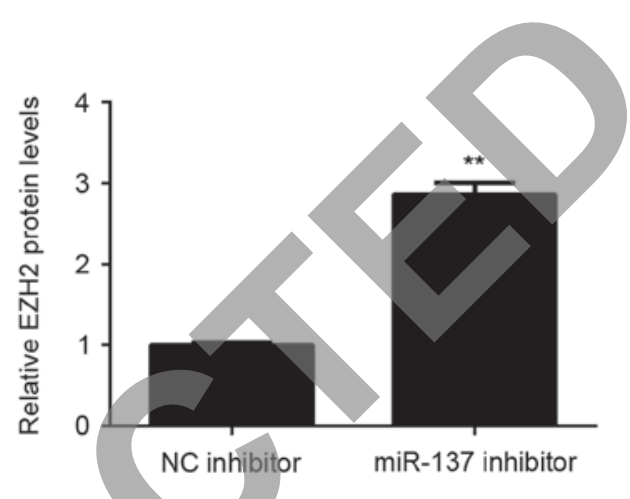

Figure 4. Effect of miR-137 mimic and inhibitor on EZH2 protein expression. (A) Western blot analysis was used to examine the protein expression of EZH2 in HepG2 cells transfected with miR-137 mimic or miR-NC. ${ }^{* *} \mathrm{P}<0.01$ vs. miR-NC. (B) HepG2 cells were transfected with miR-137 inhibitor or NC inhibitor, and reverse transcription-quantitative polymerase chain reaction was used to examine miR-137 expression. ${ }^{* *} \mathrm{P}<0.01$ vs. NC inhibitor. (C) Western blot was used to examine the protein expression of EZH2. ${ }^{* *} \mathrm{P}<0.01$ vs. NC inhibitor. miR, microRNA; $\mathrm{NC}$, negative control; EZH2, enhancer of zeste 2 polycomb repressive complex 2 subunit.

with the miR-137 levels. To further confirm the targeting relationship between miR-137 and EZH2, the expression levels of EZH2 in HCC tissues were examined. As presented in Fig. 5A, RT-qPCR data demonstrated that the mRNA levels of EZH2 were significantly upregulated in $\mathrm{HCC}$ tissues compared to adjacent non-tumor tissues. Furthermore, its expression levels were higher in HCC at high stage (III-IV) when compared with those in HCC at low stage (I-II; Fig. 5B). Further investigation demonstrated an inverse correlation between the miR-137 and EZH2 mRNA levels in HCC tissues (Fig. 5C). Therefore, these data further support that downregulation of miR-137 causes the upregulation of its target gene EZH2 in HCC. In addition, the mRNA and protein levels of EZH2 were also upregulated in the liver cell lines compared with THLE-3 cells (Fig. 5D-E).

Overexpression of EZH2 eliminates the inhibitory effects of miR-137 on the malignant phenotypes of HepG2 cells. Subsequently, the role of EZH2 in the miR-137-mediated malignant phenotypes of HCC cells was investigated. HepG2 cells were transfected with miR-137 mimic, or co-transfected with miR-137 mimic and pcDNA3.1-EZH2 ORF plasmid. RT-qPCR and western blot data indicated that the mRNA and protein levels of EZH2 were significantly upregulated in cells co-transfected with miR-137 mimic and pcDNA3.1-EZH2 ORF plasmid, when compared with those in the miR-137 group (Fig. 6A and B). Cell proliferation, migration and invasion were also examined. As indicated in Fig. 6C-E, the proliferation, invasion and migration of HepG2 cells were significantly increased in the miR-137 +
EZH2 group compared with the miR-137 group. Therefore, overexpression of EZH2 may reverse some of the inhibitory effects of miR-137 on the malignant phenotypes of HepG2 cells. These findings suggested that miR-137 inhibits cell proliferation, migration and invasion, at least partially, by directly targeting EZH2.

\section{Discussion}

The regulatory mechanism of miR-137 underlying HCC growth and metastasis remains largely unclear. In the present study, it was demonstrated that miR-137 was significantly downregulated in HCC tissues and several liver cancer cell lines. Low expression of miR-137 was significantly associated with vein invasion, lymph node metastasis, advanced clinical stage and as poor prognosis. Overexpression of miR-137 led to a significant reduction in HepG2 cell proliferation, migration and invasion. EZH2, significantly upregulated in HCC, was further identified as a direct target gene of miR-137, and the protein expression of EZH2 was negatively regulated by miR-137 in HepG2 cells. Furthermore, overexpression of EZH2 eliminated the inhibitory effects of miR-137 on the malignant phenotypes of HepG2 cells.

miR-137 has been reported to function as a tumor suppressor in various common human cancers, including colorectal cancer (17), glioblastoma (18), gastric cancer (19), multiple myeloma (20), lung cancer (11) and papillary thyroid carcinoma (21). Recently, miR-137 was reported to have a suppressive role in HCC (15). Liu et al (14) reported that 
A

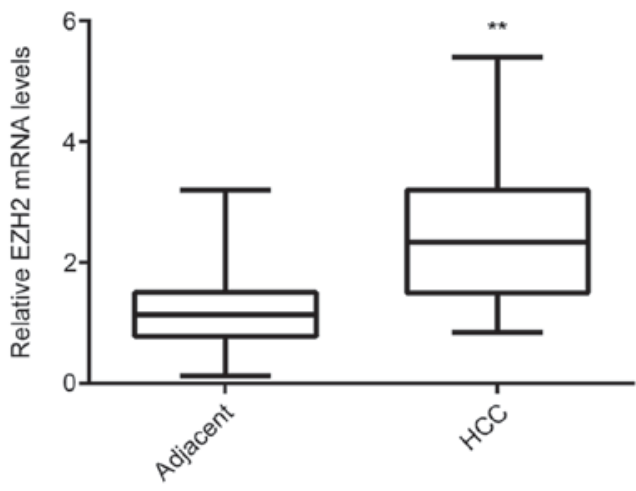

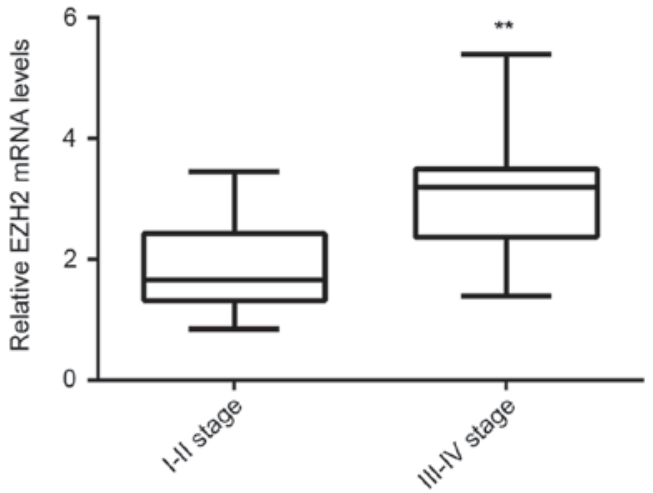

C

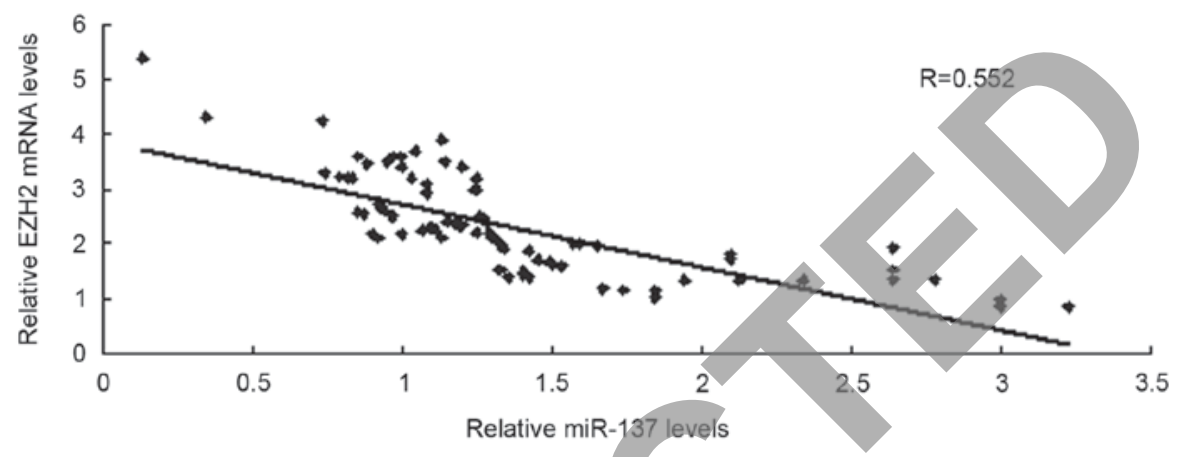

D
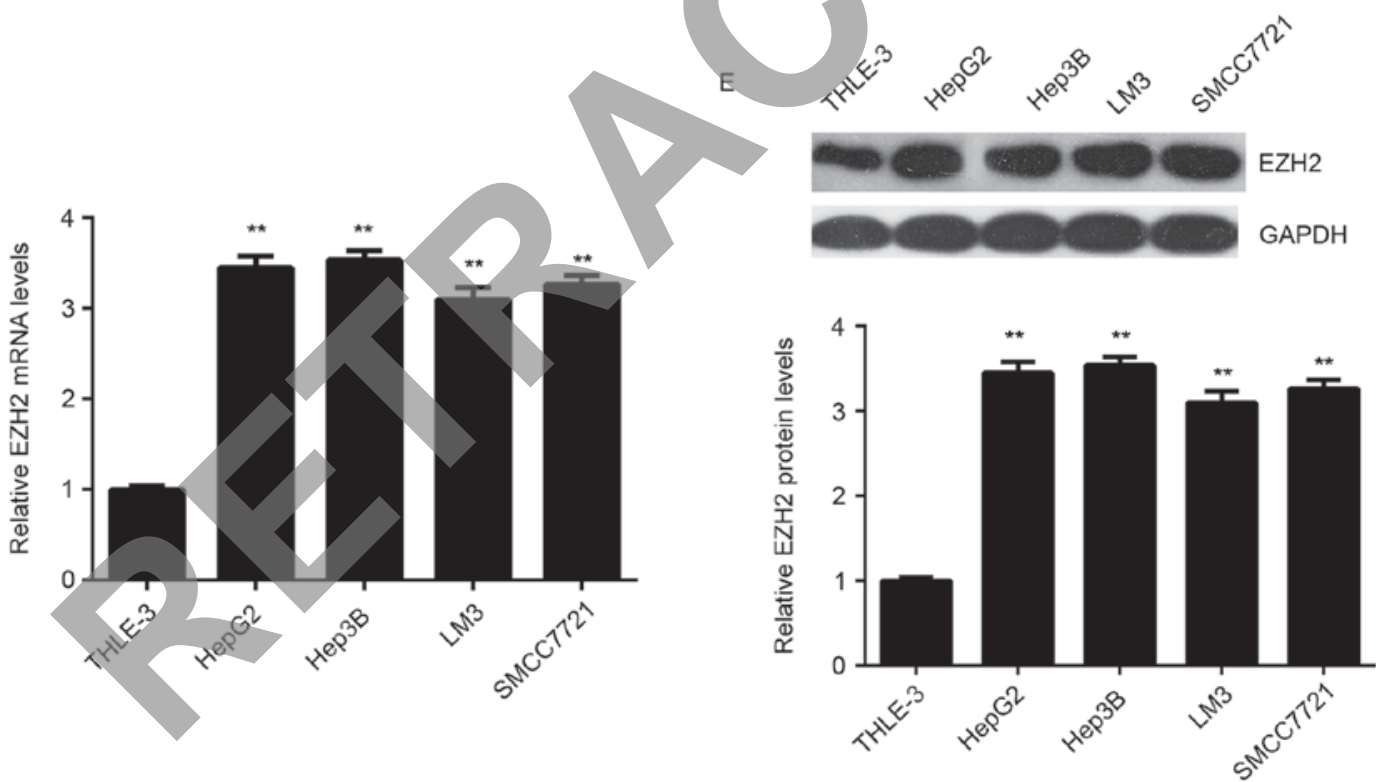

Figure 5. EZH2 expression in HCC tissues and liver cancer cell lines. (A) RT-qPCR was used to examine the EZH2 mRNA expression in HCC tissues compared with adjacent non-tumor tissues. ${ }^{* *} \mathrm{P}<0.01$ vs. adjacent. (B) RT-qPCR was used to examine the EZH2 mRNA expression in stage III-IV HCC tissues compared with stage I-II HCC tissues. ${ }^{* *} \mathrm{P}<0.01$ vs. stage I-II. (C) An inverse correlation was observed between the miR-137 and EZH2 mRNA levels in HCC tissues. (D) RT-qPCR and (E) western blot analysis were used to examine the mRNA and protein expression of EZH2 in liver cancer cell lines and THLE-3 normal liver epithelial cells. " $\mathrm{P}<0.01$ vs. THLE-3. RT-qPCR, reverse transcription-quantitative polymerase chain reaction; EZH2, enhancer of zeste 2 polycomb repressive complex 2 subunit; miR, microRNA; HCC, hepatocellular carcinoma.

miR-137 was significantly downregulated in HCC, and its decreased expression is associated with vein invasion, incomplete involucrum, and distant metastasis. In the present study, miR-137 was significantly decreased in HCC tissues compared with adjacent non-tumor tissues. Additionally, the expression of miR-137 was further lower in HCC at high stage (III-IV) compared with that in HCC at low stage (I-II). Furthermore, low expression of miR-137 was significantly associated with vein invasion, lymph node metastasis, and advanced clinical stage in HCC, but not associated with age, sex, tumor number, grade or HPV infection. Additionally, Liu et al (14) used multivariate analysis to show that miR-137 is an independent indicator for poor survival, and analysis in the current study indicated that patients with HCC with low expression of miR-137 had shorter survival time compared with those with high miR-137 levels. Data from the present study and others suggest that downregulation of miR-137 may contribute to the malignant progression of HCC. 
A

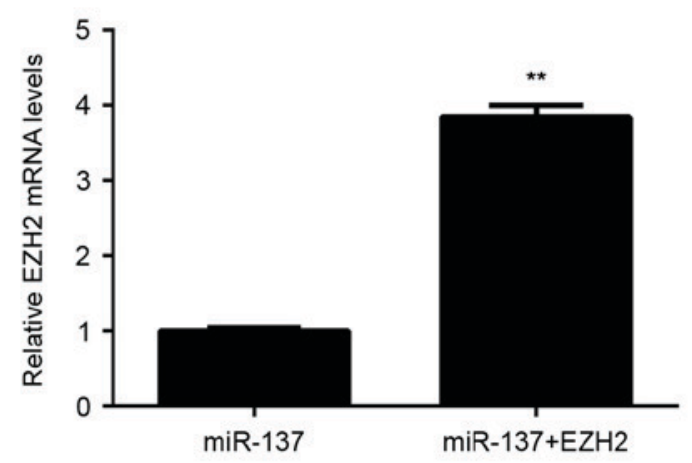

C

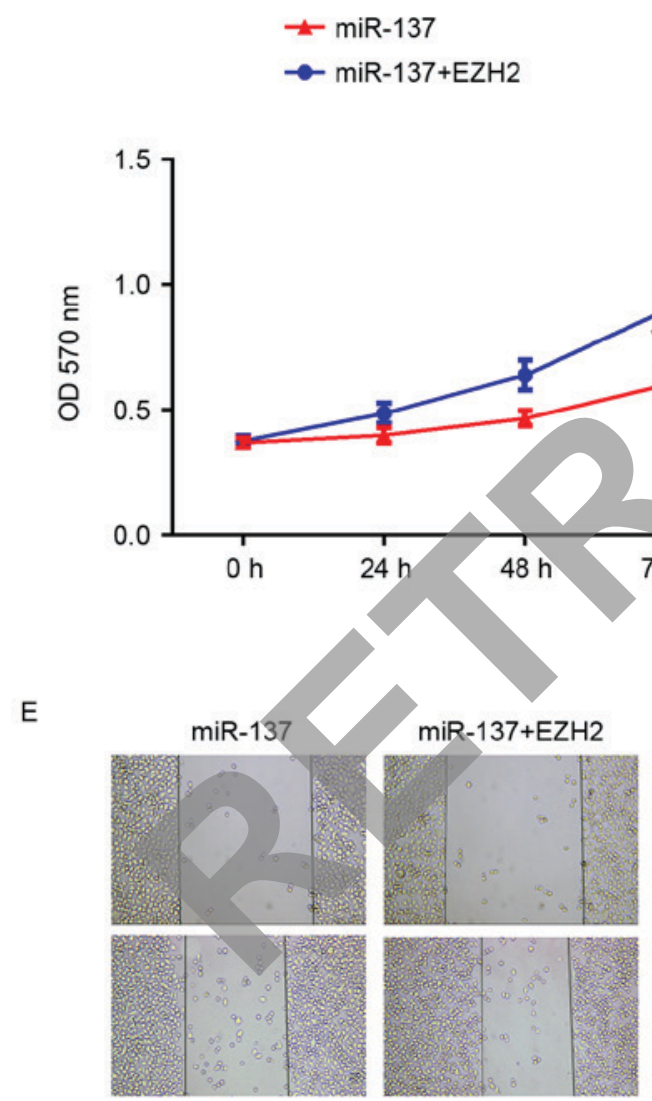

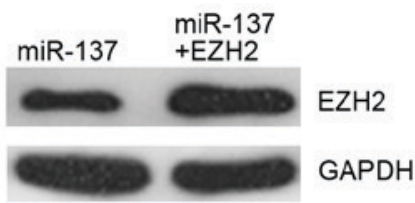

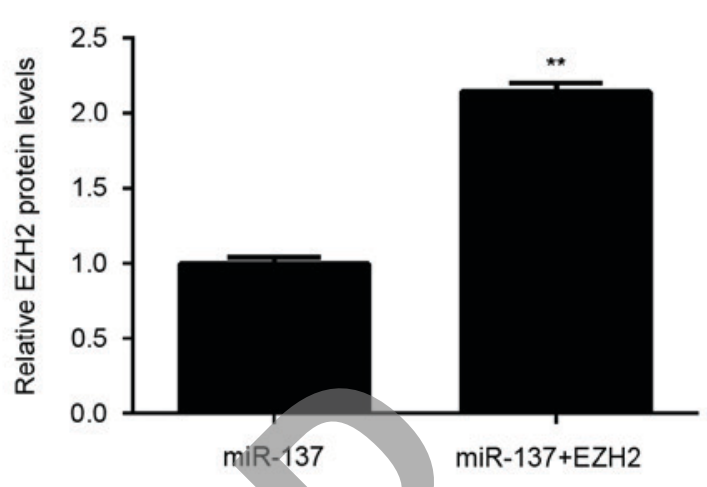

D
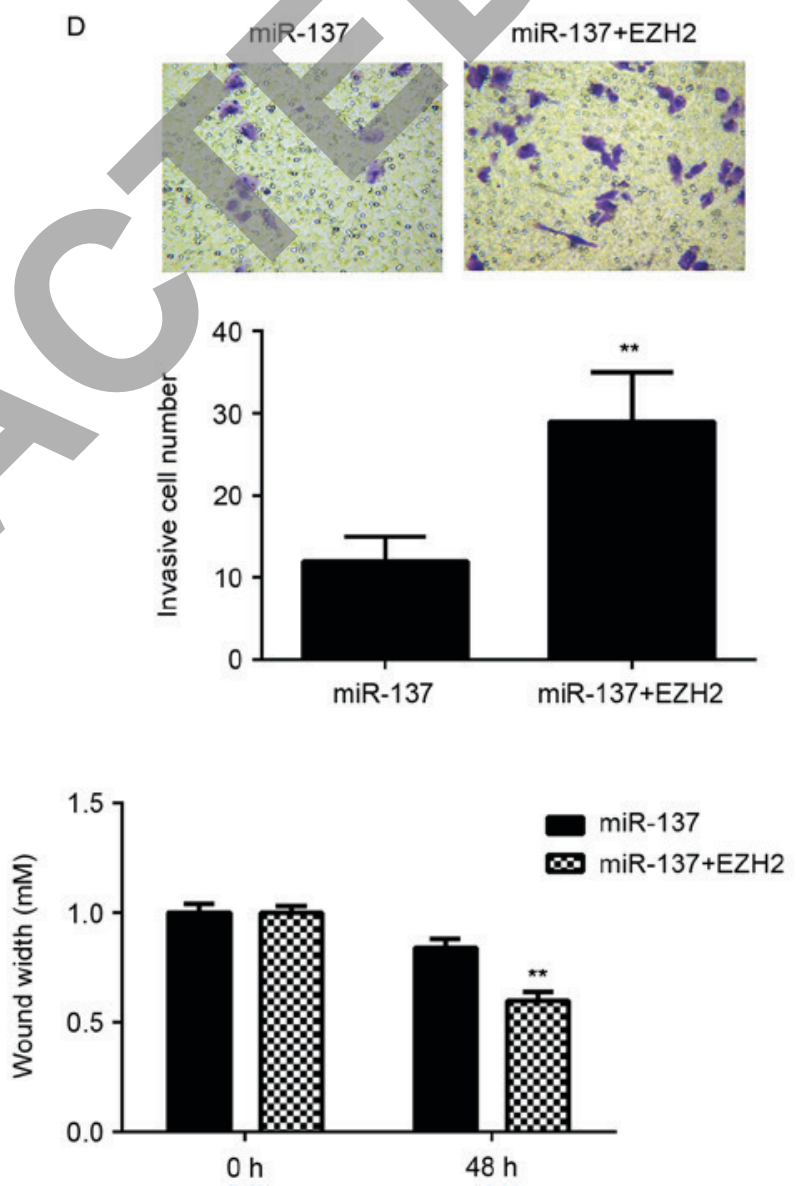

Figure 6. EZH2 overexpression rescues the effects of miR-137. HepG2 cells were transfected with miR-137 mimic or co-transfected with miR-137 mimic and EZH2 expression plasmid; (A) reverse transcription-quantitative polymerase chain reaction and (B) western blot analysis were used to examine the mRNA and protein expression of EZH2, respectively. (C) MTT assay, (D) Transwell assay and (E) wound healing assay were conducted to examine the cell proliferation, invasion and migration, respectively. ${ }^{* *} \mathrm{P}<0.01 \mathrm{vs.} \mathrm{miR-137.} \mathrm{EZH2,} \mathrm{enhancer} \mathrm{of} \mathrm{zeste} 2$ polycomb repressive complex 2 subunit; miR, microRNA.

Furthermore, in the current study miR-137 was downregulated in liver cancer cell lines compared with normal liver cells, and overexpression of miR-137 significantly decreased the proliferation, migration and invasion of HepG2 cells. Liu et al (14) also reported that miR-137 overexpression suppressed cell proliferation, migration and invasion in vitro using HCC cell lines, including SK-Hep1 and QGY-7703. Additionally, Gao et al (15) used $\mathrm{HuH7}$ and MHCC97 L HCC cell lines, and reported that upregulation of miR-137 inhibited cell proliferation and metastasis in vitro and tumor growth in vivo. These findings suggest that miR-137 has an inhibitory role in HCC growth and metastasis.

EZH2, a histone methyltransferase, is the catalytic subunit of polycomb repressive complex 2 , a highly conserved protein complex that regulates gene expression by methylating lysine 27 on histone H3 (22). EZH2 has been demonstrated to act as an 
oncogene in certain human cancers, including HCC (23-25). Sudo et al (25) reported that EZH2 was significantly upregulated in HCC tissues compared with corresponding non-tumor specimens. In the present study, EZH2 was significantly upregulated in HCC tissues and liver cancer cell lines, and its expression levels were higher in HCC at high stage (III-IV) compared low stage (I-II). Furthermore, Au et al (26) reported that the upregulation of EZH2 was significantly associated with venous invasion, direct liver invasion and absence of tumor encapsulation, and that EZH2 epigenetically silenced multiple tumor suppressor miRs, including miR-139-5p, miR-125b, miR-101, let-7c, and miR-200b, to promote liver cancer metastasis (26). In the present study, EZH2 was identified as a direct target gene of miR-137, and the protein expression of EZH2 was demonstrated to be negatively regulated by miR-137 in HepG2 cells. Additionally, an inverse correlation between the miR-137 and $\mathrm{EZH} 2$ expression levels in HCC tissues was observed. Therefore, the upregulation of EZH2 in HCC may be due to the downregulation of miR-137. Overexpression of EZH2 eliminated the suppressive effects of miR-137 on the proliferation, migration and invasion of HepG2 cells, which supports that the suppressive role of miR-137 in regulating the malignant phenotypes of HCC cells was via direct targeting of EZH2. In fact, the targeting association between miR-137 and EZH2 was also identified in several other cancers, including glioblastoma, neuroblastoma and melanoma (27-29). Luo et al (29) reported that miR-137 inhibited the invasion of melanoma cells through downregulation of multiple oncogenic target genes, including $\mathrm{EZH} 2$. Therefore, the results of the current study expand on the importance of the miR-137/EZH2 in human cancer.

In summary, the present study demonstrated that miR-137 is downregulated in HCC, and inhibited HepG2 cell proliferation, migration and invasion, at least partially, through directly targeting EZH2 oncogene. Therefore, the findings highlight the importance of miR-137/EZH2 axis in liver cancer progression.

\section{References}

1. Jemal A, Bray F, Center MM, Ferlay J, Ward E and Forman D: Global cancer statistics. CA Cancer J Clin 61: 69-90, 2011.

2. Siegel RL, Miller KD and Jemal A: Cancer statistics, 2015. CA Cancer J Clin 65: 5-29, 2015.

3. Bartel DP: MicroRNAs: Genomics, biogenesis, mechanism, and function. Cell 116: 281-297, 2004.

4. Moss EG: MicroRNAs: Hidden in the genome. Curr Biol 12 R138-R140, 2002.

5. Croce CM and Calin GA: miRNAs, cancer, and stem cell division. Cell 122: 6-7, 2005.

6. Meng F, Henson R, Wehbe-Janek H, Ghoshal K, Jacob ST and Patel T: MicroRNA-21 regulates expression of the PTEN tumor suppressor gene in human hepatocellular cancer. Gastroenterology 133: 647-658, 2007.

7. Li N, Fu H, Tie Y, Hu Z, Kong W, Wu Y and Zheng X: miR-34a inhibits migration and invasion by down-regulation of c-Met expression in human hepatocellular carcinoma cells. Cancer Lett 275: 44-53, 2009.

8. Jiang R, Deng L, Zhao L, Li X, Zhang F, Xia Y, Gao Y, Wang X and Sun B: miR-22 promotes HBV-related hepatocellular carcinoma development in males. Clin Cancer Res 17: 5593-5603, 2011

9. Dou C, Wang Y, Li C, Liu Z, Jia Y, Li Q, Yang W, Yao Y, Liu Q and Tu K: MicroRNA-212 suppresses tumor growth of human hepatocellular carcinoma by targeting FOXA1. Oncotarget 6: 13216-13228, 2015.

10. Wang CY, Zhang JJ, Hua L, Yao KH, Chen JT and Ren XQ MicroRNA-98 suppresses cell proliferation, migration and invasion by targeting collagen triple helix repeat containing 1 in hepatocellular carcinoma. Mol Med Rep 13: 2639-2644, 2016.
11. Shen H, Wang L, Ge X, Jiang CF, Shi ZM, Li DM, Liu WT, Yu X and Shu YQ: MicroRNA-137 inhibits tumor growth and sensitizes chemosensitivity to paclitaxel and cisplatin in lung cancer. Oncotarget 7: 20728-20742, 2016.

12. Liang ML, Hsieh TH, Ng KH, Tsai YN, Tsai CF, Chao ME, Liu DJ, Chu SS, Chen W, Liu YR, et al: Downregulation of miR-137 and miR-6500-3p promotes cell proliferation in pediatric high-grade gliomas. Oncotarget 7: 19723-19737, 2016.

13. Wu DC, Zhang MF, Su SG, Fang HY, Wang XH, He D, Xie YY and Liu XH: HEY2, a target of miR-137, indicates poor outcomes and promotes cell proliferation and migration in hepatocellular carcinoma. Oncotarget 7: 38052-38063, 2016.

14. Liu LL, Lu SX, Li M, Li LZ, Fu J, Hu W, Yang YZ, Luo RZ, Zhang CZ and Yun JP: FoxD3-regulated microRNA-137 suppresses tumour growth and metastasis in human hepatocellular carcinoma by targeting AKT2. Oncotarget 5: 5113-5124, 2014.

15. Gao M, Liu L, Li S, Zhang X, Chang Z and Zhang M: Inhibition of cell proliferation and metastasis of human hepatocellular carcinoma by miR-137 is regulated by CDC42. Oncol Rep 34: 2523-2532, 2015

16. Livak KJ and Schmittgen TD: Analysis of relative gene expression data using real-time quantitative PCR and the 2(-Delta Delta C (T)) method. Methods 25: 402-408, 2001.

17. Chen DL, Wang DS, Wu WJ, Zeng ZL, Luo HY, Qiu MZ, Ren C, Zhang DS, Wang ZQ, Wang FH, et al: Overexpression of paxillin induced by miR-137 suppression promotes tumor progression and metastasis in colorectal cancer. Carcinogenesis 34: 803-811, 2013.

18. Bier A, Giladi N, Kronfeld N, Lee HK, Cazacu S, Finniss S, Xiang C, Poisson L, deCarvalho AC, Slavin S, et al: MicroRNA-137 is downregulated in glioblastoma and inhibits the stemness of glioma stem cells by targeting RTVP-1. Oncotarget 4: 665-676, 2013.

9. Deng J, Lei W, Xiang X, Zhang L, Lei J, Gong Y, Song M, Wang Y, Fang Z, Yu F, et al: Cullin 4A (CUL4A), a direct target of miR-9 and miR-137, promotes gastric cancer proliferation and invasion by regulating the Hippo signaling pathway. Oncotarget 7: 10037-10050, 2016.

20. Zhang B, Ma L, Wei J, Hu J, Zhao Z, Wang Y, Chen Y and Zhao F: miR-137 suppresses the phosphorylation of AKT and improves the dexamethasone sensitivity in multiple myeloma cells via targeting MITF. Curr Cancer Drug Targets 16: 807-817, 2016.

21. Dong S, Jin M, Li Y, Ren P and Liu J: miR-137 acts as a tumor suppressor in papillary thyroid carcinoma by targeting CXCL12. Oncol Rep 35: 2151-2158, 2016

22. Italiano A: Role of the EZH2 histone methyltransferase as a therapeutic target in cancer. Pharmacol Ther 165: 26-31, 2016.

23. Zhong J, Min L, Huang H, Li L, Li D, Li J, Ma Z and Dai L: EZH2 regulates the expression of p16 in the nasopharyngeal cancer cells. Technol Cancer Res Treat 12: 269-274, 2013.

24. Zhang K, Zhang Y, Ren K, Zhao G, Yan K and Ma B: MicroRNA-101 inhibits the metastasis of osteosarcoma cells by downregulation of EZH2 expression. Oncol Rep 32: 2143-2149, 2014.

25. Sudo T, Utsunomiya T, Mimori K, Nagahara H, Ogawa K, Inoue $\mathrm{H}$, Wakiyama $\mathrm{S}$, Fujita $\mathrm{H}$, Shirouzu $\mathrm{K}$ and Mori $\mathrm{M}$ : Clinicopathological significance of EZH2 mRNA expression in patients with hepatocellular carcinoma. Br J Cancer 92: 1754-1758, 2005.

26. Au SL, Wong CC, Lee JM, Fan DN, Tsang FH, Ng IO and Wong CM: Enhancer of zeste homolog 2 epigenetically silences multiple tumor suppressor microRNAs to promote liver cancer metastasis. Hepatology 56: 622-631, 2012.

27. Sun J, Zheng G, Gu Z and Guo Z: MiR-137 inhibits proliferation and angiogenesis of human glioblastoma cells by targeting EZH2. J Neurooncol 122: 481-489, 2015.

28. Ren X, Bai X, Zhang X, Li Z, Tang L, Zhao X, Li Z, Ren Y, Wei S, Wang Q, et al: Quantitative nuclear proteomics identifies that miR-137-mediated EZH2 reduction regulates resveratrol-induced apoptosis of neuroblastoma cells. Mol Cell Proteomics 14: 316-328, 2015.

29. Luo C, Tetteh PW, Merz PR, Dickes E, Abukiwan A, Hotz-Wagenblatt A, Holland-Cunz S, Sinnberg T, Schittek B, Schadendorf D, et al: miR-137 inhibits the invasion of melanoma cells through downregulation of multiple oncogenic target genes. J Invest Dermatol 133: 768-775, 2013. 